\title{
AN APPLICATION OF FAST MULTIPOLE METHOD IN ANALYZING ACOUSTIC FILTERS
}

\author{
Chien-Ho Wu* and Chao-Nan Wang
}

\begin{abstract}
The purpose of this study is to investigate the acoustic performance of mufflers. As a numerical scheme for analyzing an acoustic filter, the boundary element method is more suitable than are domain methods, such as the finite element method, which has to mesh the domain. However, the coefficient matrices established by the boundary element method are full and often non-symmetrical. In order to decrease the computational time required, the fast multipole method has been applied. The fast multipole method, when compared with the boundary element method, reduces CPU time from an order of $N^{2}$ to $N \log ^{\gamma} N$, where $N$ is the number of unknowns and $\gamma$ is a constant. This study focused on the different geometries of plates within simple expansion acoustic filters. Numerical analysis demonstrates that the transmission loss at a particular frequency performance is better when employing a simple expansion acoustic filter with plates.
\end{abstract}

Key Words: acoustic filter, interior acoustic problem, FMM.

\section{INTRODUCTION}

An acoustic filter is a widely employed noise control tool. Many studies (Davis et al., 1954; Igarashi and Toyama, 1958; Miwa and Igarashi, 1959; Bernake, 1971; Wang, 2000) have been performed in order to predict and understand the performance of acoustic filters according to the filter theory or the transfer matrix method. Young and Crocker (1975) applied the Finite Element Method (FEM) in order to analyze the characteristics of mufflers; the results compared well to those from the plane wave theory. Panigrahi and Munjal (2005) investigated multiply connected complex acoustic filters based on network theory. Wang et al. (1998; 1999a; 1999b) analyzed three-dimensional mufflers with mean flow or absorbent material using the Boundary Element Method (BEM).

Generally, for analyzing an acoustic filter, BEM is more suitable than domain methods, such as FEM, which have to mesh the domain. However, the coefficient matrices established by BEM are full and often non-symmetrical. The CPU time is about the order of $N^{2}$ for building the matrices. To decrease

\footnotetext{
*Corresponding author. (Tel: 886-2-33665754; Email: f94525020 @ ntu.edu.tw)

The authors are with the Department of Engineering Science and Ocean Engineering, National Taiwan University, Taipei 106, Taiwan, R.O.C.
}

the computational time required, the FMM, which was recently developed, has been applied. Rokhlin (1985) developed the FMM for the 2D Laplace equation in 1985. This method was further developed by Greengard and Rokhlin (1987) for the pairwise force calculation with Coulombic or gravitational potential. The applications of FMM and related methods are found in various fields. Chen et al. $(2004 ; 2009)$ solved 2D exterior acoustic problems and successfully expanded the four kernels in the dual formulation into degenerate kernels that separate field and source points, using addition theory. Chen and Hong (1999) discussed the regularization of hypersingular integrals and divergent series based on dual boundary element methods. The FMM can also be applied to 2D seismic scattering, 3D topography and basin problems (Fujiwara, 1998; Fujiwara, 2000). The FMM for use with Laplace, Helmholtz, and heat equations was discussed by Nishimura (2002). Gomez and Power (1997) applied multipole direct and indirect BEMs to solve the 2D Stokes cavity flow.

In this study the concept of Chen and Chen (2004) will be adopted and extended to analyze the $2 \mathrm{D}$ acoustic filters. The remainder of this paper is organized as follows. The theory underlying the FMM is briefly introduced. Simple expansion acoustic filters with different rigid plates inside are then analyzed and discussed. 


\section{BASIC FORMULATION}

For an interior acoustic problem, acoustic pressure $p$ should be governed by the Helmholtz equation as follows:

$$
\nabla^{2} p+k^{2} p=0
$$

where $\nabla^{2}$ is the Laplacian operator and $k$ is the wave number. By applying the Green second identity and introducing the fundamental solution of the Helmholz equation, the boundary integral formulation can be derived as (Tanaka et al., 1985)

$$
\begin{aligned}
& C(\vec{\xi}) p(\vec{\xi}) \\
& =\int_{\Gamma} G(\vec{\xi}, \vec{s}) \frac{\partial p(\vec{s})}{\partial n(\vec{s})} d \Gamma(\vec{s}) \\
& \quad-\int_{\Gamma} \frac{\partial G(\vec{\xi}, \vec{s})}{\partial n(\vec{s})} p(\vec{s}) d \Gamma(\vec{s}), \vec{\xi} \in \Gamma,
\end{aligned}
$$

where $\vec{\xi}$ is the field point, $\vec{s}$ is the source point, $\Gamma$ is the boundary, $\partial / \partial n(\vec{s})$ is an outward normal derivative at the source point, and $C(\vec{\xi})$ is the solid angle of the field point. The free space fundamental solution of the Helmholtz equation is written in Eq. (3) and satisfies Eq. (4):

$$
G(\vec{\xi}, \vec{s})=\frac{1}{4 i} H_{0}^{(2)}(k|\vec{\xi}-\vec{s}|),
$$

where $H_{0}^{(2)}(k|\vec{\xi}-\vec{s}|)$ is the Hankel function of the second kind zeroth order, and $i$ is the square root of -1 .

$$
\nabla^{2} G(\vec{\xi}, \vec{s})+k^{2} G(\vec{\xi}, \vec{s})=-\delta(\vec{\xi}, \vec{s}),
$$

where $\delta(\vec{\xi}, \vec{s})$ is the Dirac delta function.

To solve the problem, the boundary is divided into $N$ line segments. The state variables of pressure and pressure gradient are assumed to be constant and equal to the value on each center of element $\Gamma_{\beta}$. The boundary integral formulation Eq. (2) can be rewritten as

$$
\begin{aligned}
& \frac{1}{2} p(\vec{\xi}) \\
& =\sum_{\beta=1}^{N} \int_{\Gamma_{\beta}} G(\vec{\xi}, \vec{s}) \frac{\partial p(\vec{s})}{\partial n(\vec{s})} d \Gamma_{\beta}(\vec{s}) \\
& \quad-\sum_{\beta=1}^{N} \int_{\Gamma_{\beta}} \frac{\partial G(\vec{\xi}, \vec{s})}{\partial n(\vec{s})} p(\vec{s}) d \Gamma_{\beta}(\vec{s}) .
\end{aligned}
$$

By applying Eq. (5) to each node and integrating each element, the linear algebraic system equation expressed in matrix form is

$$
[A]\{\boldsymbol{p}(\overrightarrow{\boldsymbol{s}})\}=[\boldsymbol{B}]\left\{\boldsymbol{p}_{\boldsymbol{n}}(\overrightarrow{\boldsymbol{s}})\right\},
$$

where $\{\boldsymbol{p}(\overrightarrow{\boldsymbol{s}})\}$ and $\left\{\boldsymbol{p}_{\boldsymbol{n}}(\overrightarrow{\boldsymbol{s}})\right\}=\left\{\frac{\partial \boldsymbol{p}(\overrightarrow{\boldsymbol{s}})}{\partial \boldsymbol{n}(\overrightarrow{\boldsymbol{s}})}\right\}$ are column vectors of pressure and the pressure gradient, respectively. The matrix coefficients in $[\boldsymbol{A}]$ and $[\boldsymbol{B}]$ are calculated $A_{\alpha \beta}=\frac{1}{2} \delta_{\alpha \beta}+\int_{\Gamma_{\beta}} \frac{\partial G(\vec{\xi}, \vec{s})}{\partial n(\vec{s})} d \Gamma_{\beta}$ and $B_{\alpha \beta}$ $=\int_{\Gamma_{\beta}} G(\vec{\xi}, \vec{s}) d \Gamma_{\beta}$, where subscripts $\alpha$ and $\beta$ are the labels of the collocation element and integration element, respectively. When $\alpha=\beta$, the constant $\delta_{\alpha \beta}$ $=1$; otherwise, it is zero.

\section{THE FAST MULTIPOLE METHOD}

A brief illustration of the FMM is introduced as follows. First, the kernel function $G(\vec{\xi}, \vec{s})$, which exists in the boundary integral formulation, separated the field points and source points into two terms by the addition theorem (Chen and Chen, 2004; Abramowitz and Stegun, 1965), as shown in the following equation:

$$
G(\vec{\xi}, \vec{s})=\left\{\begin{array}{l}
G^{R}=-\frac{i}{4} \sum_{m=-\infty}^{\infty} H_{m}^{(2)}(k|\vec{\xi}-\vec{\mu}|) J_{m}(k|\vec{s}-\vec{\mu}|) \cos (m \theta),|\vec{\xi}-\vec{\mu}|>|\vec{s}-\vec{\mu}| \\
G^{S}=-\frac{i}{4} \sum_{m=-\infty}^{\infty} J_{m}(k|\vec{\xi}-\vec{\mu}|) H_{m}^{(2)}(k|\vec{s}-\vec{\mu}|) \cos (m \theta),|\vec{\xi}-\vec{\mu}|<|\vec{s}-\vec{\mu}|
\end{array},\right.
$$

where $\vec{\mu}$ is the center of the multipole, $J_{m}(\zeta)$ is a Bessel function of the first kind and $\zeta$ is an argument.

Taking the normal derivative with $G(\vec{\xi}, \vec{s})$ at the source point, the function $G_{n}(\vec{\xi}, \vec{s})$ can also be derived and displayed in the following equations (Chen and Chen, 2004; Abramowitz and Stegun, 1965):

$$
G_{n}(\vec{\xi}, \vec{s})=\left\{\begin{array}{l}
G_{n}^{R}=-\frac{i}{4} \sum_{m=-\infty}^{\infty} H_{m}^{(2)}(k|\vec{\xi}-\vec{\mu}|) \frac{\partial}{\partial n(\vec{s})}\left(J_{m}(k|\vec{s}-\vec{\mu}|) \cos (m \theta)\right),|\vec{\xi}-\vec{\mu}|>|\vec{s}-\vec{\mu}| \\
G_{n}^{S}=-\frac{i}{4} \sum_{m=-\infty}^{\infty} J_{m}(k|\vec{\xi}-\vec{\mu}|) \frac{\partial}{\partial n(\vec{s})}\left(H_{m}^{(2)}(k|\vec{s}-\vec{\mu}|) \cos (m \theta)\right),|\vec{\xi}-\vec{\mu}|<|\vec{s}-\vec{\mu}|
\end{array} .\right.
$$


The partial derivatives of the Bessel, Hankel and cosine functions are displayed as follows:

$$
\begin{aligned}
& \frac{\partial}{\partial n(\vec{s})}\left(J_{m}(k|\vec{s}-\vec{\mu}|)\right. \\
& =\frac{k}{2}\left[J_{m-1}(k|\vec{s}-\vec{\mu}|)-J_{m+1}(k|\vec{s}-\vec{\mu}|)\right] \\
& \quad \cdot \frac{\left(s_{x}-\mu_{x}\right) n_{x}(\vec{s})+\left(s_{y}-\mu_{y}\right) n_{y}(\vec{s})}{|\vec{s}-\vec{\mu}|}, \\
& \frac{\partial}{\partial n(\vec{s})}\left(H_{m}^{(2)}(k|\vec{s}-\vec{\mu}|)\right. \\
& =\frac{k}{2}\left[H_{m-1}^{(2)}(k|\vec{s}-\vec{\mu}|)-H_{m+1}^{(2)}(k|\vec{s}-\vec{\mu}|)\right] \\
& \quad \cdot \frac{\left(s_{x}-\mu_{x}\right) n_{x}(\vec{s})+\left(s_{y}-\mu_{y}\right) n_{y}(\vec{s})}{|\vec{s}-\vec{\mu}|}, \\
& \frac{\partial}{\partial n(\vec{s})} \cos (m \theta)=m \frac{\sin (m \theta)}{\sin \theta}\left[a_{x} n_{x}(\vec{s})+a_{y} n_{y}(\vec{s})\right], \\
& a_{x} \\
& =\frac{\left(s_{y}-\mu_{y}\right)^{2}\left(\xi_{x}-\mu_{x}\right)-\left(s_{x}-\mu_{x}\right)\left(s_{y}-\mu_{y}\right)\left(\xi_{y}-\mu_{y}\right)}{|\vec{s}-\vec{\mu}|^{3}|\vec{\xi}-\vec{\mu}|}, \\
& =\frac{\left(s_{x}-\mu_{x}\right)^{2}\left(\xi_{y}-\mu_{y}\right)-\left(s_{x}-\mu_{x}\right)\left(s_{y}-\mu_{y}\right)\left(\xi_{x}-\mu_{x}\right)}{|\vec{s}-\vec{\mu}|^{3}|\vec{\xi}-\vec{\mu}|}, \\
& a_{y}
\end{aligned}
$$

where suffixes $x$ and $y$ are the components of the local coordinates. To simplify the expansive formulation, the origin rotates or transfers from the global coordinate to each center of the multipole as shown in Fig. 1. Therefore, the outward normal vectors of local coordinates $\left(n_{x}(\vec{s}), n_{y}(\vec{s})\right)$ are united and equal to $(0,-1)$ for an interior acoustic problem. By substituting unit outward normal vectors into Eqs. (9)(11), the component of $n_{x}(\vec{s})$ can be eliminated.

Thus, the kernel functions have been expanded into a series form. Since the kernels become singular as $\vec{s}$ approaches $\vec{\xi}$, the matrix coefficient will be calculated in two parts: integration of the regular element and singular element. This integration is discussed as follows.

\section{The Regular Integral Situation (Chen and Chen, 2004; Abramowitz and Stegun, 1965)}

In this case, the kernel functions are expanded into series form. The matrix components can be

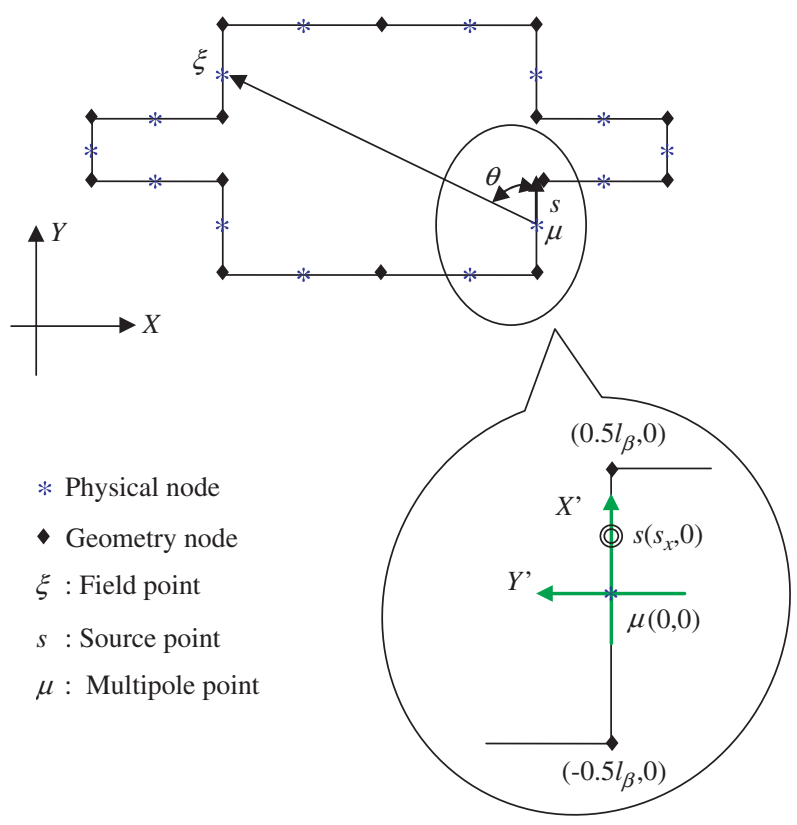

Fig. 1 The global coordinate $(X, Y)$ and local coordinate $\left(X^{\prime}, Y^{\prime}\right)$

developed using the following formula:

$$
\begin{aligned}
& B_{\alpha \beta}=\int_{\Gamma_{\beta}} G^{R} d \Gamma_{\beta} \\
& =-\frac{i}{4} \sum_{m=0}^{\infty} \varepsilon_{m} H_{2 m}^{(2)}(k|\vec{\xi}-\vec{\mu}|) \cos (2 m \theta) \\
& \cdot\left[\frac{4}{k} \sum_{n=0}^{\infty} J_{2 m+2 n+1}\left(0.5 l_{\beta} k\right)\right], \\
& \cong \sum_{m=0}^{M} C_{\alpha, \beta, m}^{1} R_{m, \beta}
\end{aligned}
$$

where $\mathrm{M}$ is a positive integer used to approximate the sums of an infinite series.

The value of $R_{m, \beta}$ is called a multipole moment, and it is related to the length of the source element. The value $C_{\alpha, \beta, m}^{1}$ is related to the field points. When the boundary is divided into uniform meshes, $R_{m, \beta}$ is calculated only once. Thus, they are displayed in the following equations:

$$
\begin{aligned}
& C_{\alpha, \beta, m}^{1}=-\frac{i}{4} \varepsilon_{m} H_{2 m}^{(2)}(k|\vec{\xi}-\vec{\mu}|) \cos (2 m \theta), \\
& R_{m, \beta} \cong \frac{4}{k} \sum_{n=0}^{M} J_{2 m+2 n+1}\left(0.5 l_{\beta} k\right),
\end{aligned}
$$

where $l_{\beta}$ is the length of the $\beta$ th source element $\Gamma_{\beta}$, and $\varepsilon_{m}$ is denoted by

$$
\varepsilon_{m}=\left\{\begin{array}{ll}
1, & m=0 \\
2, & m \neq 0
\end{array}\right\}
$$

In Eq. (14), $\theta$ is an angle from $\overrightarrow{\mu \xi}$ to $\overrightarrow{\mu s}$ as shown in 
Fig. 2. When the angle is located in the interior of the acoustic filter, such as $\theta_{1}$, the value can be calculated by

$$
\theta=\cos ^{-1}\left(\frac{(\vec{s}-\vec{\mu})(\vec{\xi}-\vec{\mu})}{|(\vec{s}-\vec{\mu})||(\vec{\xi}-\vec{\mu})|}\right) .
$$

On the contrary, when the angle is located on the exterior of the acoustic filter, such as $\theta_{2}$, the solution for the value should be amended as in the following equation:

$$
\theta=2 \pi-\cos ^{-1}\left(\frac{(\vec{s}-\vec{\mu})(\vec{\xi}-\vec{\mu})}{|(\vec{s}-\vec{\mu})||(\vec{\xi}-\vec{\mu})|}\right) .
$$

Furthermore, the value of $A_{\alpha \beta}$ can also be derived by

$$
\begin{aligned}
A_{\alpha \beta}= & \int_{\Gamma_{\beta}} G_{n}^{R} d \Gamma_{\beta}(s) \\
\cong & \frac{i k}{4} \sum_{m=0}^{M} H_{2 m+1}^{(2)}(k|(\vec{\xi}-\vec{\mu})|) \sin [(2 m+1) \theta] \\
& \cdot\left(R_{m, \beta}+R_{(m+1), \beta}\right) \\
= & \sum_{m=0}^{M} C_{\alpha, \beta, m}^{2}\left(R_{m, \beta}+R_{(m+1), \beta}\right)
\end{aligned}
$$

where the value $C_{\alpha, \beta, m}^{2}$ is defined as

$$
C_{\alpha, \beta, m}^{2}=\frac{i k}{4} H_{2 m+1}^{(2)}(k|\vec{\xi}-\vec{\mu}|) \sin [(2 m+1) \theta] .
$$

For further details of the regular integral, refer to the research of Chen and Chen (2004).

\section{The Singular Integral Situation}

In this study, the integration values for the two singular kernels are derived using the conventional BEM, which is displayed as follows. A weakly singular integral led to the limiting form for small arguments in the formula. The value of weakly singular integral can then be derived using Eq. (22):

$$
\begin{aligned}
B_{\alpha \alpha} & =\int_{\Gamma_{\beta}} G(\vec{\xi}, \vec{s}) d \Gamma_{\beta}=\frac{l_{\beta}}{8 i} \int_{-1}^{1} H_{0}^{(2)}\left(k \eta \frac{l_{\beta}}{2}\right) d \eta \\
& \cong-\frac{l_{\beta}}{4} \frac{2}{\pi} \int_{0}^{1} \ln \left(k \eta \frac{l_{\beta}}{2}\right) d \eta=-\frac{l_{\beta}}{2 \pi}\left[\ln \left(k \frac{l_{\beta}}{2}\right)-1\right] .
\end{aligned}
$$

As the normal and surface of the strongly singular integral are orthogonal, the value of the kernel can be defined as follows:

$$
A_{\alpha \alpha}=\frac{1}{2}+\int_{\Gamma_{\beta}} \frac{\partial G(\vec{\xi}, \vec{s})}{\partial n(\vec{s})} d \Gamma_{\beta}=\frac{1}{2} .
$$

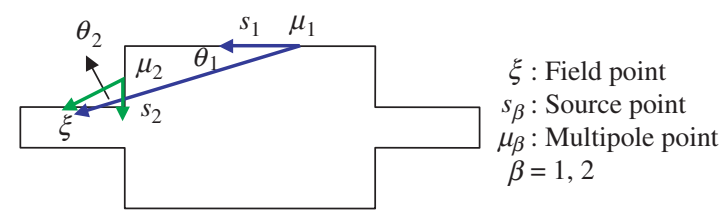

Fig. 2 The angles located in the interior/exterior of the acoustic filter

\section{TRANSMISSION LOSS}

This work investigates the characteristics of an acoustic filter using the FMM. Transmission loss for an acoustic filter is generally used as the parameter representing its performance evaluation. The derivation of transmission loss for an acoustic filter is as follows.

An acoustic system has three parts: the inlet, outlet and other, and can be simulated using a linear acoustic four-pole network (Munjal, 1987; Wang et al., 2001). By adopting the linear Euler equation, the pressure gradient can be converted to a normal velocity. Furthermore, the acoustic filter wall is assumed rigid; thus Eq. (6) becomes (Wang and Liao, 1998; Wang, 1999; Wang, 2000)

$$
\left[\begin{array}{ll}
\boldsymbol{A}_{11} & \boldsymbol{A}_{12} \\
\boldsymbol{A}_{21} & \boldsymbol{A}_{22}
\end{array}\right]\left\{\begin{array}{c}
\boldsymbol{p}_{\text {in, out }} \\
\boldsymbol{p}_{\text {other }}
\end{array}\right\}=(-i k)\left[\begin{array}{l}
\boldsymbol{B}_{11} \\
\boldsymbol{B}_{21}
\end{array}\right]\left\{\rho c u_{\boldsymbol{n}}\right\}_{\text {in, out }},
$$

where $\rho c$ is the characteristic impedance of the medium and $u_{n}$ is normal velocity. We assume that only the plane wave was propagated in the inlet and outlet pipes. The relation of pressure and particle velocity between the inlet and outlet of an acoustic linear filter can then be represented by (Young and Crocker, 1975)

$$
\left\{\begin{array}{c}
\boldsymbol{p} \\
\boldsymbol{\rho c u _ { n }}
\end{array}\right\}_{\text {in }}=\left[\begin{array}{ll}
\boldsymbol{T}_{11} & \boldsymbol{T}_{12} \\
\boldsymbol{T}_{21} & \boldsymbol{T}_{22}
\end{array}\right]\left\{\begin{array}{c}
\boldsymbol{p} \\
\boldsymbol{\rho c} u_{n}
\end{array}\right\}_{\text {out }}
$$

where $T_{11}, T_{12}, T_{21}$ and $T_{22}$ are known as the fourpole parameters.

We assume that $p_{\text {in }}=1$ and $u_{n, \text { out }}=0$ in Eq. (25); thus $T_{11}$ and $T_{21}$ of the transfer matrix can be derived. Similarly $T_{21}$ and $T_{22}$ can also be obtained by assigning $u_{n, \text { in }}=1$ and $p_{\text {out }}=0$

$$
T_{11}=\left.\frac{p_{\text {in }}}{p_{\text {out }}}\right|_{u_{n, \text { out }}=0}, T_{21}=\left.\frac{-\rho c u_{n, \text { in }}}{p_{\text {out }}}\right|_{u_{n, \text { out }}=0},
$$

$$
T_{12}=\left.\frac{p_{\text {in }}}{\rho c u_{n, \text { out }}}\right|_{p_{\text {out }}=0}, T_{22}=\left.\frac{-\rho c u_{n, \text { in }}}{\rho c u_{n, \text { out }}}\right|_{p_{\text {out }}=0} .
$$




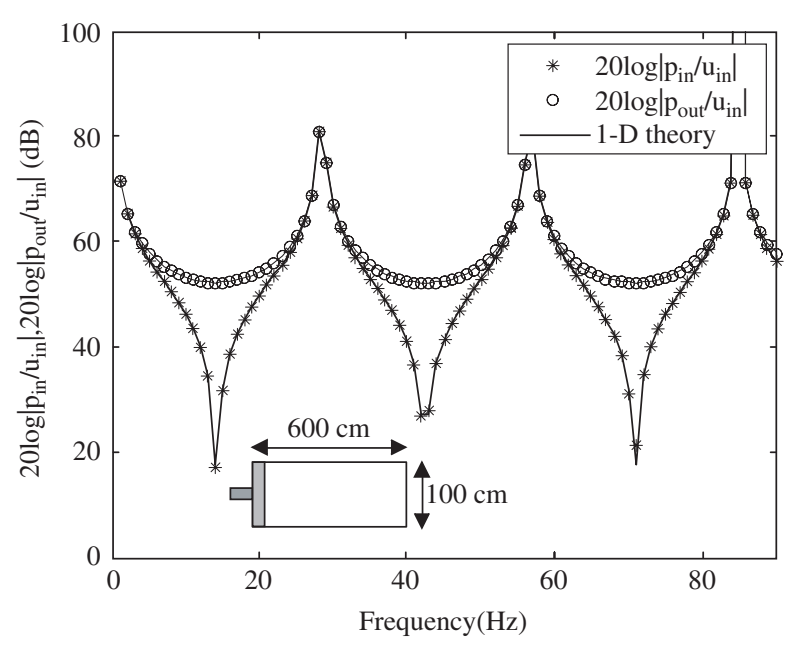

Fig. 3 The acoustic pressures of the straight-duct

The transmission loss of an acoustic filter is defined as the difference between input sound power level and transmitted sound power level. Sound power can be derived based on the product of the cross sectional area and intensity. According to the derivation in (Munjal, 1987), the ratio of input power and transmitted power can be obtained and related to the fourpole parameters in Eq. (30). Therefore, transmission loss of an acoustic filter in terms of the four-pole parameters can be expressed as (Young and Crocker, 1975; Munjal, 1987; Panigrahi and Munjal, 2005)

$$
\begin{aligned}
T L & =10 \log _{10}\left(\frac{W_{i}}{W_{t}}\right) \\
& =10 \log _{10}\left(\frac{S_{\text {in }}}{S_{\text {out }}}\left|\frac{T_{11}+T_{12}+T_{21}+T_{22}}{2}\right|^{2}\right),
\end{aligned}
$$

where $W_{i}$ is incident sound power, $W_{t}$ is transmitted sound power and $\frac{S_{\text {in }}}{S_{\text {out }}}$ denotes the ratio of inlet to outlet cross-sectional area.

\section{NUMERICAL RESULTS}

To verify the efficacy of the present method, a rigid straight duct with a piston at the duct end is analyzed (Tanaka et al., 1985). Notably, $\frac{p_{\text {in }}}{u_{\text {in }}}$ and $\frac{p_{\text {out }}}{u_{\text {in }}}$ can be calculated by the FMM. Fig. 3 presents the results of a rigid straight duct with a piston at the duct end, where $u_{i n}$ is the mean value of particle velocities for all of the elements on the inlet boundary, and $p_{\text {in }}$ and $p_{\text {out }}$ are the mean values of pressure at the inlet and outlet boundaries, respectively. The solid lines in Fig. 3 are defined by Eqs. (31) and (32), which are based on plane wave theory:

$$
\frac{p_{i n}}{u_{\text {in }}}=-i \rho c \frac{1}{\tan k l},
$$

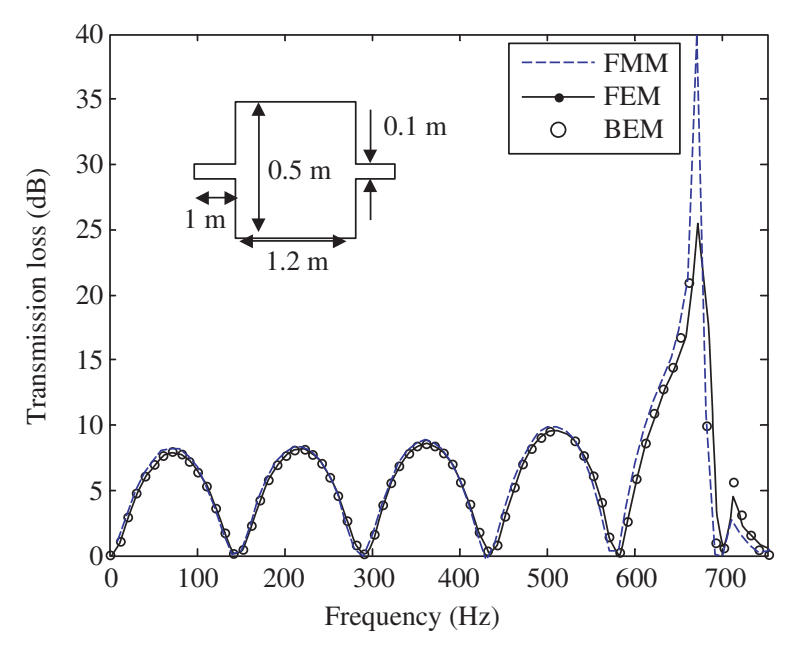

Fig. 4 Transmission loss of a simple expansion acoustic filter compared with the results of Bilawchuk et al.

$$
\frac{p_{\text {out }}}{u_{\text {in }}}=-i \rho c \frac{1}{\sin k l}
$$

where $l$ is the straight duct length, $\rho$ is density of the medium (density of air is $1.2 \mathrm{~kg} / \mathrm{m}^{3}$ ) and $c$ is the speed of sound $(c=340 \mathrm{~m} / \mathrm{s})$. The agreement between FMM and $1 \mathrm{D}$ theory is good.

Figure 4 presents the geometry and transmission loss of a simple expansion acoustic filter. A comparison of the FMM, FEM and BEM indicates that the results were generally the same. The results of FEM and BEM were obtained from the research of Bilawchuk and Fyfe (2003). These cases demonstrate in detail that the FMM prediction is reliable; therefore, this method can be expanded to other cases.

Figure 5(a) shows the geometry and transmission loss of a simple expansion acoustic filter. The agreement between FMM and BEM is good. Since kernel functions are expanded into the infinite series, discussing the convergence is useful. If too many terms are required, the efficiency of the proposed method will decrease. The absolute error is defined as

$$
\text { Error }=\left|T L_{F M M}-T L_{B E M}\right|,
$$

where $T L_{B E M}$ and $T L_{F M M}$ are the transmission loss indicated by the BEM and FMM, respectively. Since the constant element is considered in this study, and a wavelength must include about six to eight nodes for the spatial size in acoustic fields, Fig. 5(b) exhibits the absolute error of the transmission loss against different frequencies. It stands to reason that the absolute error is less than 0.2 when the constant $M$ is equal to 6 or 7 and that it can be tolerated.

For the simple expansion acoustic filter case (Fig. 5), Fig. 6 shows the absolute error of the transmission loss versus the Mth partial sum in approximating the infinite series in Eq. (14) and Eq. (20). 


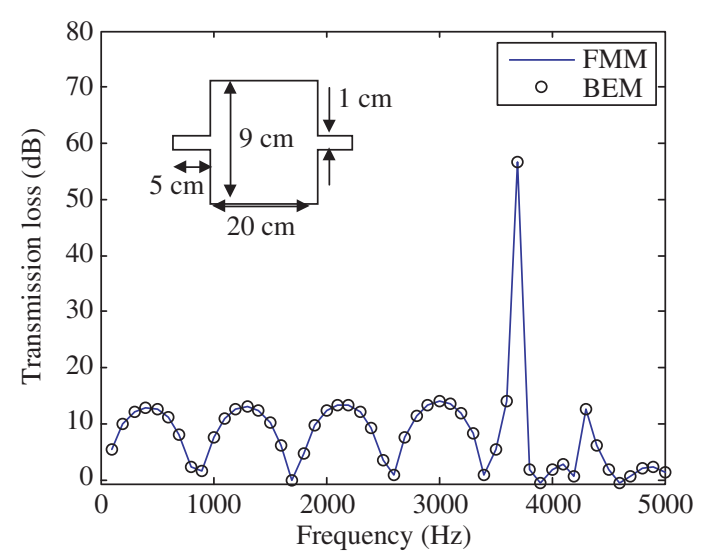

(a)
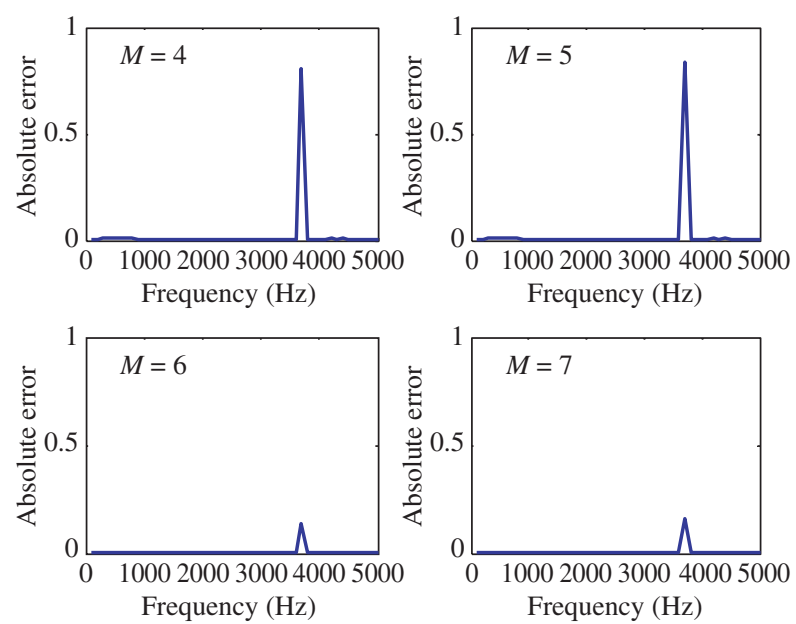

(b)

Fig. 5 (a) Transmission loss of a simple expansion acoustic filter $(N=234, M=6)(b)$ The absolute error of the transmission loss $(N=234)$

Fig. 6(a) shows the sum of the infinite series at a frequency of $100 \mathrm{~Hz}$. For boundary meshes of 78, 156 and 234 elements, seven terms are sufficient to approximate the sum of the infinite series in order to generate the required accuracy. Fig. 6(b) presents a similar analysis for $5000 \mathrm{~Hz}$ with 156, 234 and 312 elements. Again, partial sum of only seven terms can generate accurate results. Thus, the summation index is chosen from 0-6, i.e., seven terms, in all the following numerical analyses which are used to approximate the infinite series.

The most important characteristics of the FMM are that it accelerates the speed of the required calculation. To illustrate this characteristic, the same acoustic filter (Fig. 5) is utilized. Fig. 7 presents the required CPU times for different mesh numbers using the FMM and BEM at a frequency of $100 \mathrm{~Hz}$. Fig. 7 indicates that the FMM reduces CPU time from the order of $\frac{N^{2}}{3100}$ to $\frac{N}{3500} \log _{2.5} N$. This analytical result demonstrates that the FMM obviously reduces computing time.

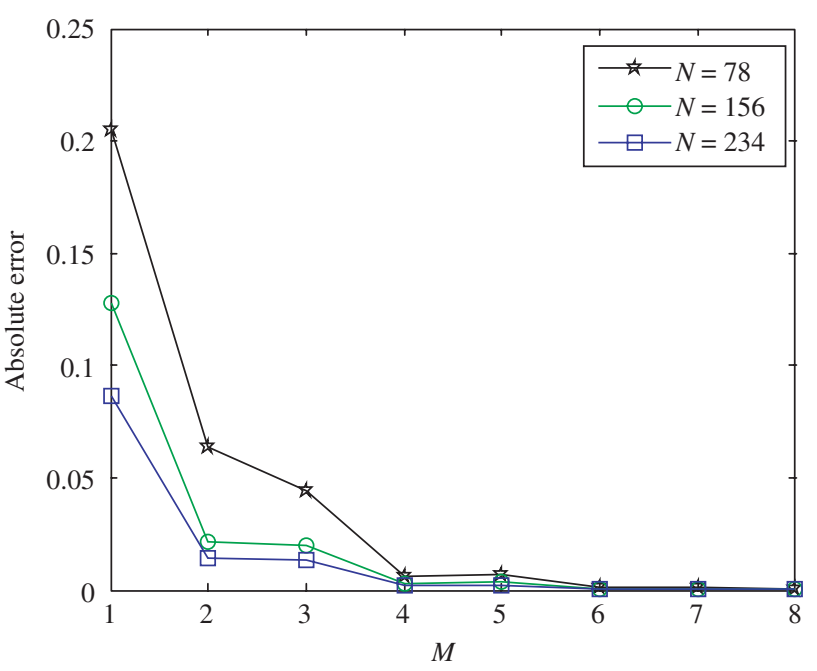

(a)

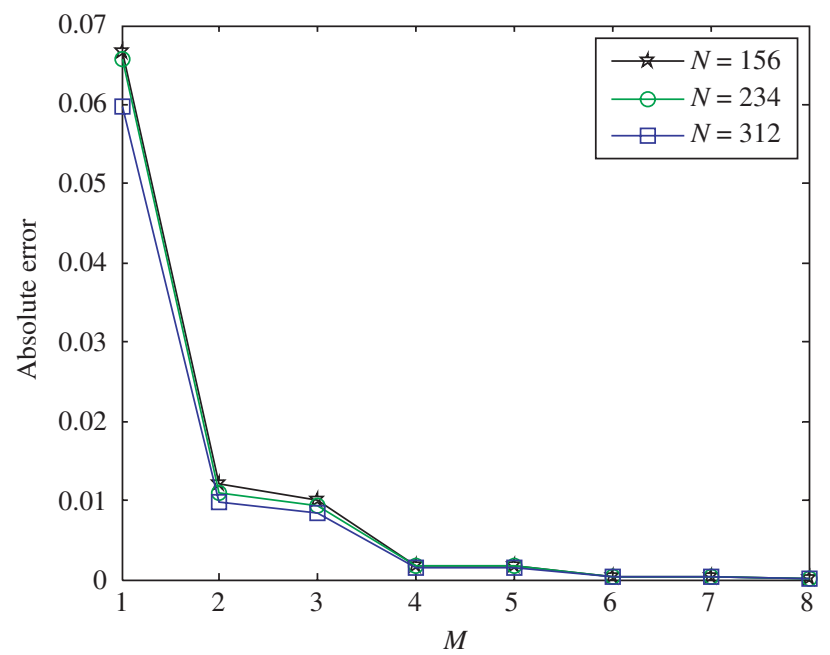

(b)

Fig. 6 The absolute error of the transmission loss versus the Mth partial sums with different number of elements (a) Frequency of $100 \mathrm{~Hz}$ (b) Frequency of $5000 \mathrm{~Hz}$

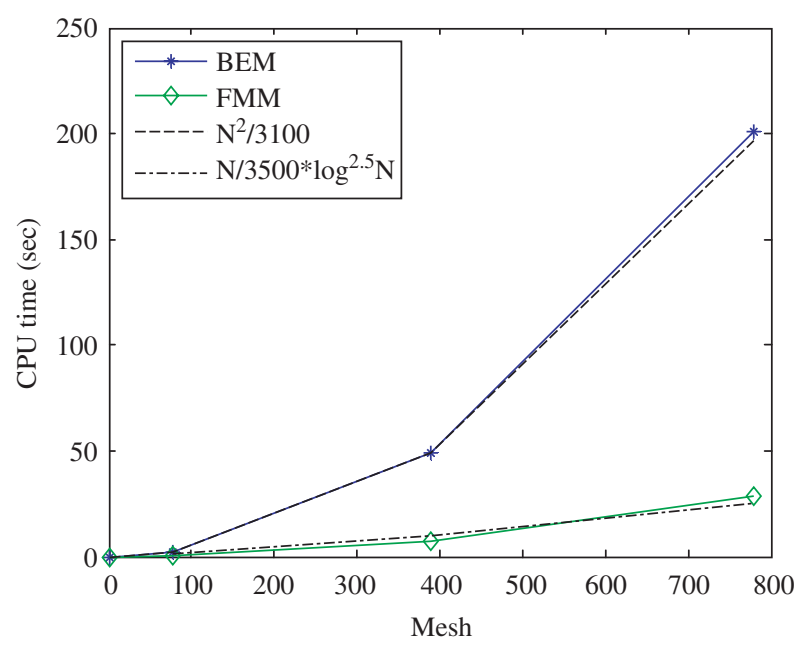

Fig. 7 CPU time versus the number of elements $N$ for the simple expansion acoustic filter 


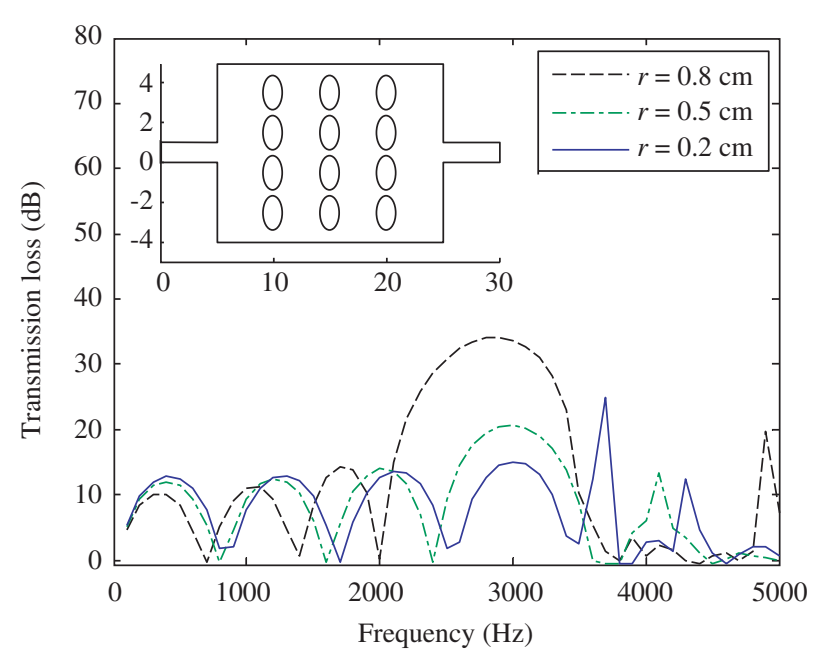

Fig. 8 Transmission loss of an acoustic filter with rigid circular cylinders

Next, this study explores the phenomena of an acoustic filter with rigid circular cylinders. Fig. 8 displays the geometry of a simple expansion acoustic filter with circular cylinders, and its transmission loss. The radii of the circular cylinders are $0.2,0.5$ and $0.8 \mathrm{~cm}$, respectively. The dimensions of the simple expansion acoustic filter are the same as those in Fig. 5 and are used in the following cases. The circular cylinders are uniformly located inside the simple expansion acoustic filter. The noise is reduced better for the acoustic filter when the rigid circular cylinders are larger. Additionally, the transmission loss of the acoustic filter with largest circular cylinders has better efficiency with frequencies at about 2000-3500 Hz in this design.

This study now analyzes acoustic filters with rigid saw-toothed plates. Fig. 9 shows the geometry of the plate and the numerical results for rigid sawtoothed plates with heights of $\mathrm{h} 1$ of $0.4,0.6$ and 0.8 $\mathrm{cm}$. The analytical result indicates that the transmission loss of a simple expansion with saw-toothed plates at about $2500-3500 \mathrm{~Hz}$ is better than in the case of a simple expansion acoustic filter (Fig. 5). Incidentally, the performance can be improved by increasing the height of $h 1$.

This study now extends the observation of transmission loss to an acoustic filter with a rigid plate. Fig. 10 shows the geometry of the simple expansion acoustic filter with rigid plate, and demonstrates its performance. As Fig. 10 indicates, the height of h2 strongly affects the capacity of an acoustic filter. In this case, the highest plate has the best performance at roughly $1500-3700 \mathrm{~Hz}$. Furthermore, an acoustic filter with a rigid plate (Fig. 10) with approximately 1500-3500 Hz has finer performance in comparison with a simple expansion acoustic filter (Fig. 5). The

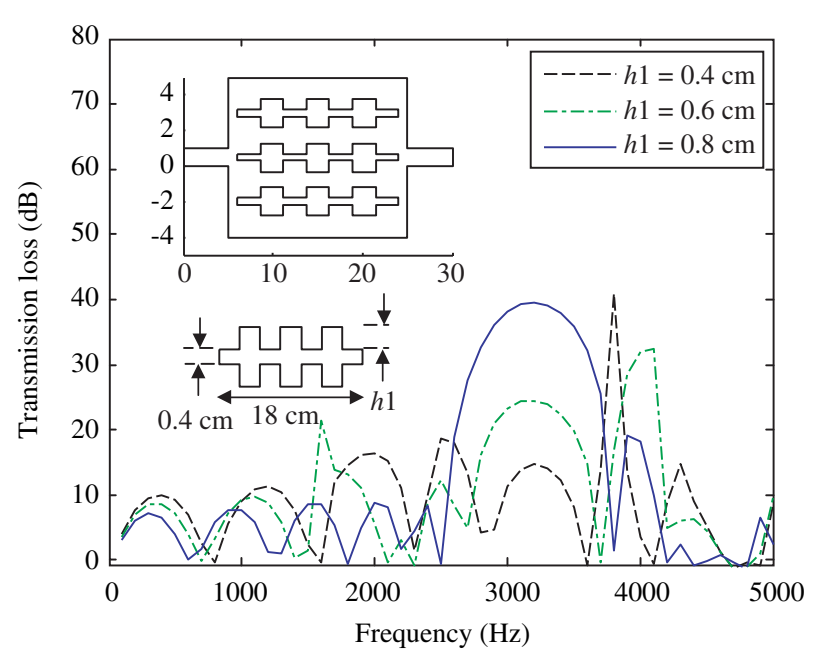

Fig. 9 The transmission loss of a simple expansion acoustic filter with rigid saw-toothed plates

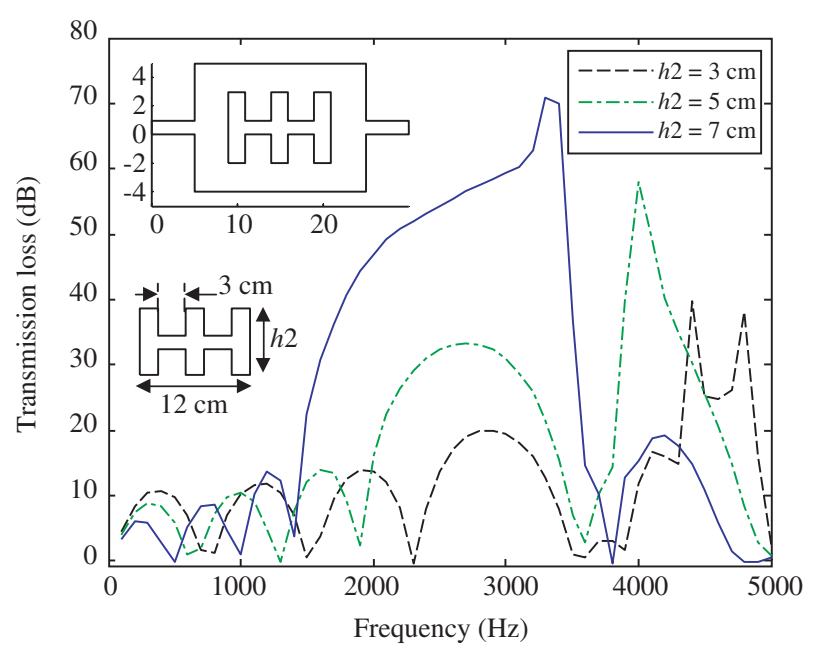

Fig. 10 The transmission loss of the acoustic filter with a rigid plate

reason for the improvement is that the rigid plate acts as a resonator in order to reduce the sound energy at specific frequencies; therefore, better resonant frequencies can be obtained.

\section{CONCLUSIONS}

It is concluded, from what has been said above, that the FMM improves the efficiency of the conventional BEM in analyzing performance of an acoustic filter. The fundamental solution is expanded into a series form. When the boundary is divided into uniform meshes, the integration of the source may be obtained only once. The FMM can reduce CPU time to the order $N \log ^{\gamma} N$ by reason of the separated technique. When compared with different methods, such as BEM, FEM and plane wave theory, the agreement is good. 
An acoustic filter with different rigid plates inside has been analyzed and discussed. Simple expansion of the acoustic filter with rigid circular cylinders, sawtoothed plates, or plates improves the noise reduction at specific frequencies. This study extended the FMM to analyze 2D acoustic filters; this extension was shown to be feasible. In future work, the FMM could be applied to $3 \mathrm{D}$ problems.

\section{NOMENCLATURE}

$\boldsymbol{A}, \boldsymbol{B}$
$c$
$C(\vec{\xi})$
$C_{\alpha, \beta, m}^{1}, C_{\alpha, \beta, m}^{2}$
$G$
$G_{n}$
$i$
$k$
$l_{\beta}$
$m$
$M$
$n_{x}$
$n_{y}$
$N$
$p$
$p_{n}$
$R_{m, \beta}$
$s$
$S_{\text {in }}$
$S_{\text {out }}$
$T_{11}, T_{12}, T_{21}$ and $T_{22}$
$u_{n}$
$W_{i}$
$W_{t}$
$\alpha$

$\beta$

$\delta$

$\varepsilon_{m}$

$\Gamma$

$\gamma$

$\mu$

$\theta$

$\xi$

$\rho$

$\zeta$

\section{REFERENCES}

Abramowitz, M., and Stegun, I. A., 1965, "Handbook of Mathematical Functions with Formulas, Graphs and Mathematical Tables," Dover, NY, USA.

Bernake, L. L., 1971, Noise and Vibration Control, McGraw-Hill, NY, USA.

Bilawchuk, S., and Fyfe, K. R., 2003, "Comparison and Implementation of the Various Numerical Methods Used for Calculating Transmission Loss in Silencer Systems," Applied Acoustics, Vol. 64, No. 9, pp. 903-916.

Chen, J. T., and Chen, K. H., 2004, “Applications of the Dual Integral Formulation in Conjunction with Fast Multipole Method in Large-scale Problems for 2D Exterior Acoustics," Engineering Analysis with Boundary Elements, Vol. 28, No. 6, pp. 685-709.

Chen, J. T., and Hong, H. K., 1999, "Review of Dual Boundary Element Methods with Emphasis on Hypersingular Integrals and Divergent Series," Applied Mechanics Reviews, American Society of Mechanical Engineers, Vol. 52, No. 1, pp. 1733.

Chen, K. H., Chen, J. T., Kao, J. H., and Lee, Y. T., 2009, “Applications of Dual Integral Formulation in Conjunction with Fast Mutipole Method to Oblique Incident Wave Problem," International Journal for Numerical Methods in Fluids, Vol. 59, No. 7, pp. 711-751.

Davis, D. D., Stokes, J. M., Moore, D., and Steven, L., 1954, "Theoretical and Experimental Investigation of Mufflers with Comments on Engine Exhaust Muffler Design," Report No. 1192, National Advisory Committee for Aeronautics.

Fujiwara, H., 1998, "The Fast Multipole Method for Integral Equation of Seismic Scattering Problems," Geophysical Journal International, Vol. 133, No. 3, pp. 773-782.

Fujiwara, H., 2000, “The Fast Multipole Method for Solving Integral Equations of Three-dimensional Topography and Basin Problems," Geophysical Journal International, Vol. 140, No. 1, pp. 198210 .

Gomez, J. E., and Power, H., 1997, “A Multipole Direct and Indirect BEM for 2D Cavity Flow at Low Reynolds Number," Engineering Analysis with Boundary Elements, Vol. 19, No. 1, pp. 17-31.

Greengard, L., and Rokhlin, V., 1987, "A Fast Algorithm for Particle Simulations," Journal of Computational Physics, Vol. 73, No. 2, pp. 325-348.

Igarashi, J., and Toyama, M., 1958, "Fundamentals of Acoustical Silencers, Part 1: Theory and Experiment of Acoustic Low-pass Filter," Report No. 339, Aeronaut Research Institute University of Tokyo, pp. 233-241.

Miwa, T., and Igarashi, J., 1959, "Fundamentals of Acoustical Silencers, Part 2: Determination of Four Terminal Constants of Acoustical Element," Report No. 344, Aeronaut Research Institute University of 
Tokyo, pp. 67-85.

Munjal, M. L., 1987, Acoustics of Ducts and Mufflers, John Wiley \& Sons, NY, USA.

Nishimura, N., 2002, "Fast Multipole Accelerated Boundary Integral Equation Methods," Applied Mechanics Review, American Society of Mechanical Engineers, Vol. 55, No. 4, pp. 299-324.

Panigrahi, S. N., and Munjal, M. L., 2005, "Plane Wave Propagation in Generalized Multiply Connected Acoustic Filters," The Journal of the Acoustical Society of America, Vol. 118, No. 5, pp. 2860-2868.

Rokhlin, V., 1985, "Rapid Solution of Integral Equations of Classical Potential Theory," Journal of Computational Physics, Vol. 60, No. 2, pp. 187207.

Tanaka, T., Fujikawa, T., Abe, T., and Utsuno, H., 1985, "A Method for the Analytical Prediction of Insertion Loss of a Two-dimensional Muffler Model Based on the Transfer Matrix Derived from the Boundary Element Method," Journal of Vibration, Acoustics, Stress, and Reliability in Design, Vol. 107, No. 1, pp. 86-91.

Wang, C. N., 1999a, "A Numerical Analysis for Perforated Muffler Components with Mean Flow," Journal of Vibration and Acoustics, Vol. 121, No.
2, pp. 231-236.

Wang, C. N., 1999b, "Numerical Decoupling Analysis of a Resonator with Absorbent Material," Applied Acoustics, Vol. 58, No. 2, pp. 109-122.

Wang, C. N., 2000, "A Boundary Element Analysis for Simple Expansion Silencers with Mean Flow," Journal of the Chinese Institute of Engineers, Vol. 23, No. 4, pp. 529-536.

Wang, C. N., and Liao, C. Y., 1998, "Boundary Integral Equation Method for Evaluating the Performance of Straight-through Resonator with Mean Flow," Journal of Sound and Vibration, Vol. 216, No. 2, pp. 281-294.

Wang, C. N., Chen, Y. N., and Tsai J. Y., 2001, "The Application of Boundary Element Evaluation on a Silencer in the Presence of a Linear Temperature Gradient," Applied Acoustics, Vol. 62, No. 6, pp. 707-716.

Young, C. I. J., and Crocker, M. J., 1975, "Prediction of Transmission Loss in Mufflers by the Finiteelement Method," The Journal of the Acoustical Society of America, Vol. 57, No. 1, pp. 144-148.

Manuscript Received: Sep. 23, 2008

Revision Received: Feb. 27, 2009

and Accepted: Mar. 31, 2009 